\title{
Transamination Reactions with Multiple Turnovers Catalyzed by Hydrophobic \\ Pyridoxamine Cofactors in the Presence of Polyethylenimine Polymers
}

Lei Liu, Wenjun Zhou, Jason Chruma, and Ronald Breslow

\section{Supporting Information}

\section{Synthesis}

a. Chemicals: Solvents, inorganic salts, and organic reagents were purchased from commercial resources and used without further purification unless otherwise mentioned. 2-Amino-2-phenyl-propionic acid, purchased from Aldrich Co., was purified by preparative RP-HPLC with a Vydac Protein \& Peptide C18 column.

b. Chromatography: Merck pre-coated $0.25 \mathrm{~mm}$ silica plates containing a $254 \mathrm{~nm}$ fluorescence indicator were used for analytical thin-layer chromatography. Flash chromatography was performed on 230-400 mesh silica (Silica Gel 60) from EM Science. The size-exclusion chromatography was performed using Sephadex ${ }^{\circledR G 10, ~ G 15, ~ G 25, ~}$ G50, and G75 from Pharmacia Biochemtech. Analytical HPLC was run on a HP1090 liquid chromatography (series II) equipped with a DR5 pumping system, a temperaturecontrolled autosampler, and a diode-array UV-vis detector. Vydac protein \& peptide C18 reverse phase analytical columns (particle size $5 \mu \mathrm{m}, 4.6 \times 150 \mathrm{~mm}$ ) were used as solid phase.

c. Spectroscopy: NMR spectra were obtained on a Bruker DPX 300 or $400 \mathrm{MHz}$ spectrometer. UV-vis spectra were taken on a Varian Cary IE UV-vis spectrometer. CI MS spectra were taken on a Nermag R-10-10 instrument. FAB MS spectra were taken on a JEOL JMS-DX-303 HF instrument using either glycerol or p-nitrobenzyl alcohol as matrices. Matrix assisted laser desorption ionization (MALDI) mass spectra were acquired using a Bruker Biflex-III time-of-flight mass spectrometer. Positive ion mass spectra were acquired in linear mode, and the ions were generated by using a nitrogen laser $(337 \mathrm{~nm})$ pulsed at $3 \mathrm{~Hz}$ with a pulse width of 3 nanoseconds. Ions were accelerated at 19-20,000 volts and amplified using a discrete dynode multiplier. Spectra (100 to 200) were summed into a LeCroy LSA1000 high speed signal digitizer. All data processing was performed using Bruker XMass/XTOF V 5.0.2. 
d. Compound synthesis and characterization:

\section{4-Aminomethyl-2-methyl-5-propylsulfanylmethyl-pyridin-3-ol:}

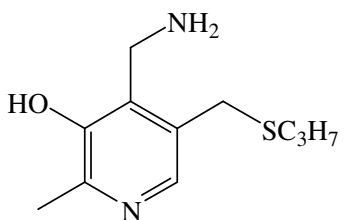

$0.7 \mathrm{ml}$ of 1-propanethiol was dissolved in $10 \mathrm{ml}$ of methanol. $10 \mathrm{ml}$ of an aqueous ammonia solution (37\%) was added. After the mixture was stirred for 10 minutes, $0.3 \mathrm{~g}$ of 4-aminomethyl-5-bromomethyl-2-methyl-pyridin-3-ol dibromide was added. The solution was stirred for 30 minutes. The solvent was removed. The product was purified using preparative RP-HPLC. CI MS: $227.4(\mathrm{M}+1) .{ }^{1} \mathrm{H}$ NMR $\left(\mathrm{CD}_{3} \mathrm{OD}, 300 \mathrm{MHz}\right): 8.03$, s, $1 \mathrm{H} ; 4.32$, s, 2H; 3.87, s, 2H; 2.60, s, 3H; 2.43, t, 2H, J = 7.6 Hz; 1.61, m, 2H; 0.97, t, 3H, $\mathrm{J}=7.3 \mathrm{~Hz}$. UV-vis: $323 \mathrm{~nm}(\mathrm{pH}=7.5)$.

\section{4-Aminomethyl-5-hexylsulfanylmethyl-2-methyl-pyridin-3-ol:}<smiles>Cc1ncc(CSc2ccccc2)c(CN)c1O</smiles>

$0.6 \mathrm{ml}$ of 1-hexanethiol was dissolved in $10 \mathrm{ml}$ of methanol. $10 \mathrm{ml}$ of ammonia aqueous solution (37\%) was added. After the mixture was stirred for 10 minutes, $0.3 \mathrm{~g}$ of 4aminomethyl-5-bromomethyl-2-methyl-pyridin-3-ol dibromide was added. The solution was stirred for 30 minutes. The solvent was removed. The product was purified using preparative RP-HPLC. CI MS: $269.6(\mathrm{M}+1) .{ }^{1} \mathrm{H}$ NMR $\left(\mathrm{CD}_{3} \mathrm{OD}, 300 \mathrm{MHz}\right): 8.13, \mathrm{~s}, 1 \mathrm{H}$; 4.51, s, 2H; 4.02, s, 2H; 2.85, s, 3H; 2.67, t, 2H, J = 7.3 Hz; 1.75-1.35, m, 8H; 1.01, t, 3H, $\mathrm{J}=6.9 \mathrm{~Hz}$. UV-vis: $324 \mathrm{~nm}(\mathrm{pH}=7.5)$.

\section{Thioacetic acid S-nonyl ester:}

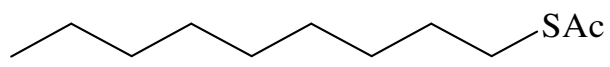

$2 \mathrm{~g}$ of 1-nonylbromide was added to $100 \mathrm{ml}$ of DMF. $5 \mathrm{~g}$ of KSAc was added. The reaction was stirred at $70{ }^{\circ} \mathrm{C}$ for 8 hours. The solvent was removed. The product was purified using flash chromatography. ${ }^{1} \mathrm{H} \mathrm{NMR}\left(\mathrm{CDCl}_{3}, 300 \mathrm{MHz}\right): 3.03, \mathrm{t}, 2 \mathrm{H}, \mathrm{J}=7.3 \mathrm{~Hz}$; 2.47, s, 3H; 1.83-1.33, m, 14H; 1.03, t, 3H, J = 6.9 Hz. CI MS: $203.4(\mathrm{M}+1)$.

\section{4-Aminomethyl-2-methyl-5-nonylsulfanylmethyl-pyridin-3-ol:}


<smiles>Cc1ncc(C[AsH2])c(CN)c1O</smiles>

$0.5 \mathrm{~g}$ of thioacetic acid S-nonyl ester was dissolved in $20 \mathrm{ml}$ of ethanol. $0.9 \mathrm{ml}$ of EtONa (21\% solution in EtOH) was added. After 10 minutes, $0.2 \mathrm{~g}$ of 4-aminomethyl-5bromomethyl-2-methyl-pyridin-3-ol dibromide was added. The solution was stirred for 30 minutes. The solvent was removed. The product was purified using preparative RPHPLC. CI MS: $311.7(\mathrm{M}+1) .{ }^{1} \mathrm{H}$ NMR $\left(\mathrm{CD}_{3} \mathrm{OD}, 300 \mathrm{MHz}\right): 7.60, \mathrm{~s}, 1 \mathrm{H} ; 4.73, \mathrm{~s}, 2 \mathrm{H} ; 3.90$, $\mathrm{s}, 2 \mathrm{H} ; 2.55, \mathrm{~m}, 5 \mathrm{H} ; 1.75-1.31, \mathrm{~m}, 14 \mathrm{H} ; 1.03$, t, 3H, J = 7.0 Hz. UV-vis: $324 \mathrm{~nm}(\mathrm{pH}=$ $7.5)$.

\section{4-Aminomethyl-5-dodecylsulfanylmethyl-2-methyl-pyridin-3-ol:}

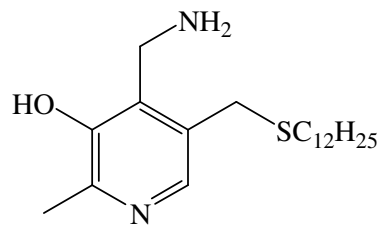

$0.6 \mathrm{ml}$ of 1-dodecanethiol was dissolved in $10 \mathrm{ml}$ of methanol. $10 \mathrm{ml}$ of ammonia aqueous solution (37\%) was added. After the mixture was stirred for 10 minutes, $0.3 \mathrm{~g}$ of 4-aminomethyl-5-bromomethyl-2-methyl-pyridin-3-ol dibromide was added. The solution was stirred for 30 minutes. The solvent was removed. The product was purified using preparative RP-HPLC. CI MS: $353(\mathrm{M}+1)$. ${ }^{1} \mathrm{H}$ NMR (CD $\left.{ }_{3} \mathrm{OD}\right): 8.21, \mathrm{~s}, 1 \mathrm{H} ; 4.45$, s, $2 \mathrm{H} ; 4.01, \mathrm{~s}, 2 \mathrm{H} ; 2.71, \mathrm{~s}, 3 \mathrm{H} ; 2.53$, t, 2H; 1.63, m, 2H; 1.40, m, 18H; 0.93, t, 3H. UV-vis: $324 \mathrm{~nm}(\mathrm{pH}=7.5)$.

\section{3-[2-(Bis-decyl-carbamoyl)-ethyldisulfanyl]-N,N-bis-decyl-propionamide:}

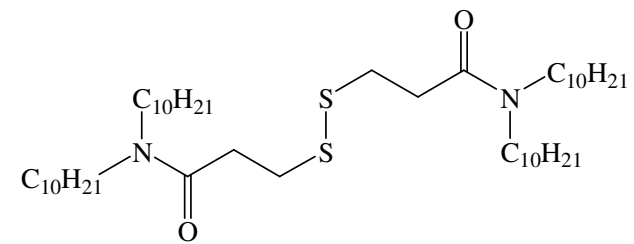

$2.1 \mathrm{~g}$ of 3-(2-carboxy-ethyldisulfanyl)-propionic acid was dissolved in $20 \mathrm{ml}$ of benzene. $10 \mathrm{ml}$ of $\mathrm{SOCl}_{2}$ was added. The reaction was refluxed for 2 hours, after which the solution became clear. Then the solvent was removed by distillation. The resulting acid

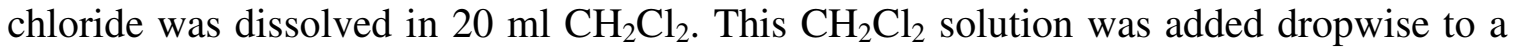
flask containing $20 \mathrm{ml}$ of $\mathrm{CH}_{2} \mathrm{Cl}_{2}, 6.0 \mathrm{~g}$ of $\mathrm{NH}\left(\mathrm{C}_{10} \mathrm{H}_{21}\right)_{2}$, and $3.0 \mathrm{ml} \mathrm{Et}_{3} \mathrm{~N}$ at $0{ }^{\circ} \mathrm{C}$. The reaction was stirred overnight. The product was obtained after the solution was washed 
with aqueous $\mathrm{HCl}$ solution and aqueous $\mathrm{NaOH}$ solution. $\mathrm{CI} \mathrm{MS}: 770(\mathrm{M}+1) .{ }^{1} \mathrm{H} \mathrm{NMR}$ $\left(\mathrm{CDCl}_{3}\right): 3.34, \mathrm{~m}, 8 \mathrm{H} ; 3.05, \mathrm{t}, 4 \mathrm{H}, \mathrm{J}=7.1 \mathrm{~Hz} ; 2.81, \mathrm{t}, 4 \mathrm{H} ; 1.65, \mathrm{~m}, 8 \mathrm{H} ; 1.40, \mathrm{~m}, 56 \mathrm{H}$; $0.98, \mathrm{t}, 12 \mathrm{H}$.

3-(4-Aminomethyl-5-hydroxy-6-methyl-pyridin-3-ylmethylsulfanyl)-N,N-bis-decylpropionamide:

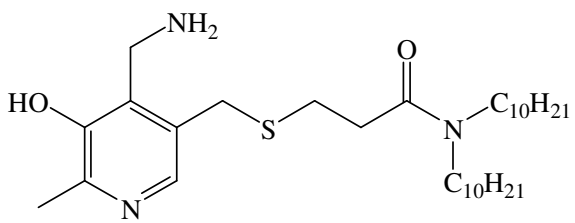

$0.5 \mathrm{~g}$ of 3-[2-(bis-decyl-carbamoyl)-ethyldisulfanyl]-N,N-bis-decyl-propionamide was dissolved in $10 \mathrm{ml}$ of ethanol. $0.4 \mathrm{~g}$ of $\mathrm{NaBH}_{4}$ was added and the mixture was stirred for 20 minutes. Then $0.2 \mathrm{~g}$ of 4-aminomethyl-5-bromomethyl-2-methyl-pyridin-3-ol dibromide was added. The reaction was stirred for 1 hour. The product was purified using preparative reversed-phase HPLC. ${ }^{1} \mathrm{H}$ NMR $\left(\mathrm{CD}_{3} \mathrm{OD}, 300 \mathrm{MHz}\right): 7.91, \mathrm{~s}, 1 \mathrm{H} ; 4.41$, s, 2H; 3.99, s, 2H; 2.85, m, 4H; 2.66, s, 3H; 1.59, m, 32H; 1.05, t, 6H, J = 6.8 Hz. CI MS: 536.6 $(\mathrm{M}+1)$. UV-vis: $323 \mathrm{~nm}\left(\mathrm{CH}_{3} \mathrm{OH}: \mathrm{H}_{2} \mathrm{O}=1: 1, \mathrm{pH}=7.5\right)$.

\section{4-(3-Hydroxy-10a,11b-dimethyl-hexadecahydro-cyclopenta[b]phenanthren-10-yl)-} pentanoic acid methyl ester:

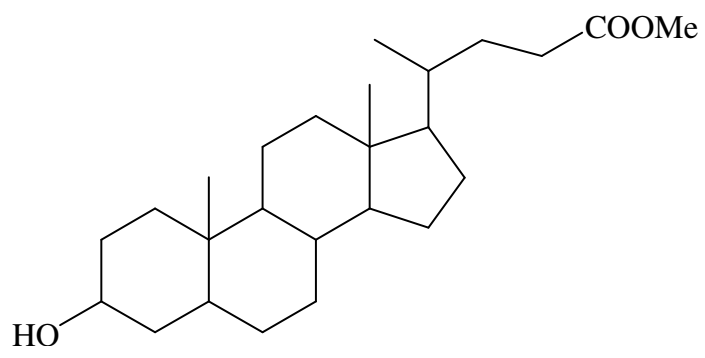

$2.5 \mathrm{~g}$ of lithocholic acid was dissolved in $25 \mathrm{ml}$ of DMF. $0.30 \mathrm{~g}$ of $\mathrm{NaH}$ was added. Then $5 \mathrm{ml}$ of $\mathrm{CH}_{3} \mathrm{I}$ was added. The reaction was stirred at room temperature for 5 hours. The solvent was removed. The product was purified using chromatography. CI MS: 373.6 $\left(\mathrm{M}+1-\mathrm{H}_{2} \mathrm{O}\right) .{ }^{1} \mathrm{H} \mathrm{NMR}\left(\mathrm{CDCl}_{3}, 300 \mathrm{MHz}\right): 3.80, \mathrm{~m}, 4 \mathrm{H} ; 2.41, \mathrm{~m}, 2 \mathrm{H} ; 0.79, \mathrm{~s}, 3 \mathrm{H}$.

10-(4-Hydroxy-1-methyl-butyl)-10a,11b-dimethyl-hexadecahydro-cyclopenta[b]phenanthren-3-ol: 


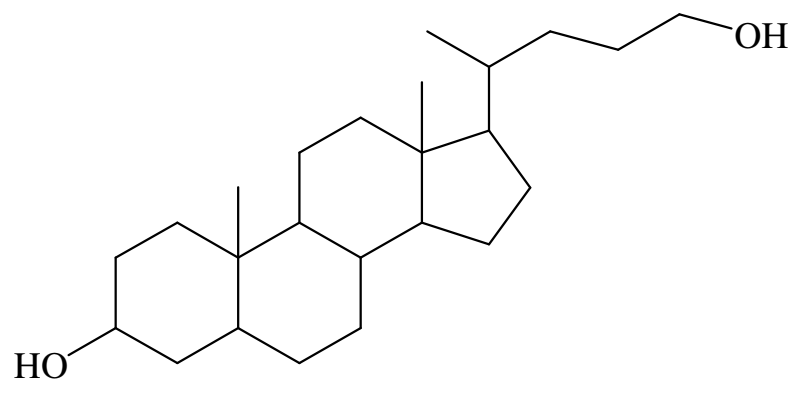

$2.1 \mathrm{~g}$ of 4-(3-hydroxy-10a,11b-dimethyl-hexadecahydro-cyclopenta[b]phenanthren-10yl)-pentanoic acid methyl ester was dissolved in $30 \mathrm{ml}$ of THF. At zero degree, $10 \mathrm{ml}$ of Red-Al solution in toluene was added. The reaction was stirred at room temperature for 3 hours. The reaction was quenched by $\mathrm{MeOH}$. The product was purified using flash chromatography. CI MS: $345\left(\mathrm{M}+1-\mathrm{H}_{2} \mathrm{O}\right) .{ }^{1} \mathrm{H}$ NMR $\left(\mathrm{CDCl}_{3}, 300 \mathrm{MHz}\right): 3.73, \mathrm{~m}, 3 \mathrm{H}$; $0.79, \mathrm{~s}, 3 \mathrm{H}$.

Toluene-4-sulfonic acid 4-(3-hydroxy-10a,11b-dimethyl-hexadecahydro-cyclopenta[b]phenanthren-10-yl)-pentyl ester:

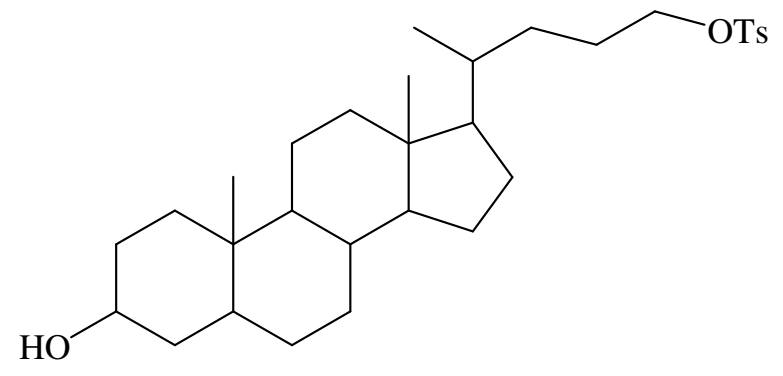

$2.0 \mathrm{~g}$ of 10-(4-hydroxy-1-methyl-butyl)-10a,11b-dimethyl-hexadecahydro-cyclopenta[b]phenanthren-3-ol was dissolved in $30 \mathrm{ml}$ of $\mathrm{CH}_{2} \mathrm{Cl}_{2}$. At zero degree, $0.77 \mathrm{ml}$ of triethylamine was added. Then $1.1 \mathrm{~g}$ of $\mathrm{TsCl}$ was added. The reaction was stirred at room temperature for 2 days. The product was purified using flash chromatography. CI MS: $499.8\left(\mathrm{M}+1-\mathrm{H}_{2} \mathrm{O}\right) .{ }^{1} \mathrm{H} \mathrm{NMR}\left(\mathrm{CDCl}_{3}, 300 \mathrm{MHz}\right): 7.83, \mathrm{~d}, 2 \mathrm{H}, \mathrm{J}=8.3 \mathrm{~Hz} ; 7.52, \mathrm{~d}, 2 \mathrm{H} ; 4.11$, t, $2 \mathrm{H}, \mathrm{J}=7.3 \mathrm{~Hz} ; 3.73, \mathrm{~m}, 1 \mathrm{H} ; 2.57$, s, 3H; 0.75, s, 3H.

Thioacetic acid S-[4-(3-hydroxy-10a,11b-dimethyl-hexadecahydro-cyclopenta[b]phenanthren-10-yl)-pentyl] ester:<smiles>CC(C)CCCC(C)C1CCC2C3CCC4CC(O)CCC4(C)C3CCC12C</smiles> 
$1.2 \mathrm{~g}$ of toluene-4-sulfonic acid 4-(3-hydroxy-10a,11b-dimethyl-hexadecahydrocyclopenta-[b]phenanthren-10-yl)-pentyl ester was dissolved in $30 \mathrm{ml}$ of DMF. $5 \mathrm{~g}$ of KSAc was added. The reaction was stirred at $70{ }^{\circ} \mathrm{C}$ for 5 hours. The product was purified using flash chromatography. CI MS: $404\left(\mathrm{M}+1-\mathrm{H}_{2} \mathrm{O}\right) .{ }^{1} \mathrm{H} \mathrm{NMR}\left(\mathrm{CDCl}_{3}, 300 \mathrm{MHz}\right): 3.71$, m, 1H; 2.99, m, 2H; 2.41, s, 3H; 0.72, s, 3H.

\section{4-Aminomethyl-5-[4-(3-hydroxy-10a,11b-dimethyl-hexadecahydro-cyclopenta[b]- phenanthren-10-yl)-pentylsulfanylmethyl]-2-methyl-pyridin-3-ol:}

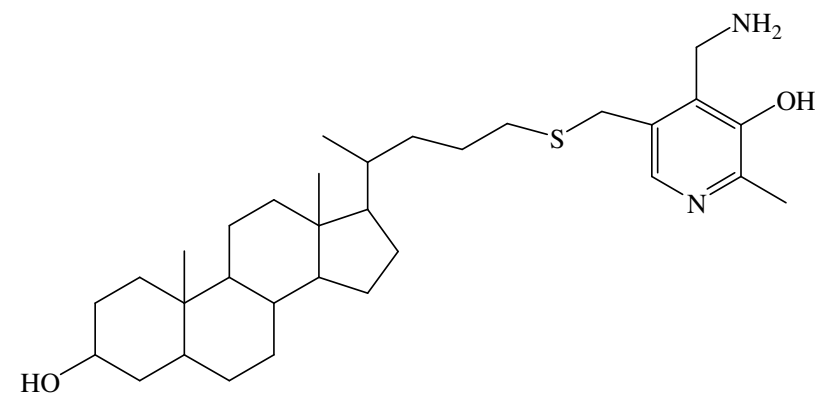

$0.2 \mathrm{~g}$ of thioacetic acid S-[4-(3-hydroxy-10a,11b-dimethyl-hexadecahydro-cyclopenta[b]phenanthren-10-yl)-pentyl] ester was dissolved in $10 \mathrm{ml}$ of EtOH. $0.5 \mathrm{ml}$ of EtONa $(21 \%$ solution in EtOH) was added. After the mixture was stirred for 10 minutes, $0.3 \mathrm{~g}$ of 4 aminomethyl-5-bromomethyl-2-methyl-pyridin-3-ol dibromide was added. The solution was stirred for 30 minutes. The solvent was removed. The product was purified using preparative HPLC. CI MS: $530.2(\mathrm{M}+1) .{ }^{1} \mathrm{H}$ NMR (CD $\left.\mathrm{OD}, 300 \mathrm{MHz}\right): 7.71, \mathrm{~s}, 1 \mathrm{H} ; 4.36$, $\mathrm{s}, 2 \mathrm{H} ; 3.81, \mathrm{~s}, 2 \mathrm{H} ; 3.75, \mathrm{~m}, 1 \mathrm{H} ; 2.55, \mathrm{~m}, 5 \mathrm{H} ; 0.81$, s, 3H. UV-vis: $322 \mathrm{~nm}(\mathrm{pH}=7.5)$.

\section{4-(tert-Butoxycarbonylamino-methyl)-3-hydroxy-5-hydroxymethyl-1,2-dimethyl- pyridinium:}

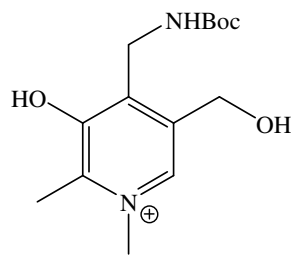

$0.35 \mathrm{~g}$ of (3-hydroxy-5-hydroxymethyl-2-methyl-pyridin-4-ylmethyl)-carbamic acid tertbutyl ester was dissolved in $15 \mathrm{ml}$ of acetonitrile. $5 \mathrm{ml}$ of MeI was added. The reaction was refluxed for 1 hour. The solid product was collected after filtration. CI MS: 283.2 $(\mathrm{M}+) .{ }^{1} \mathrm{H}$ NMR $\left(\mathrm{D}_{2} \mathrm{O}, 300 \mathrm{MHz}\right): 8.31, \mathrm{~s}, 1 \mathrm{H} ; 5.07, \mathrm{~s}, 2 \mathrm{H} ; 4.49, \mathrm{~s}, 2 \mathrm{H} ; 4.28, \mathrm{~s}, 3 \mathrm{H} ; 2.72$, s, $3 \mathrm{H} ; 1.50, \mathrm{~s}, 9 \mathrm{H}$.

\section{4-Aminomethyl-5-bromomethyl-3-hydroxy-1,2-dimethyl-pyridinium:}




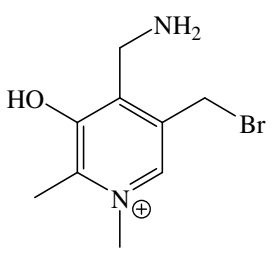

$0.4 \mathrm{~g}$ of 4-(tert-butoxycarbonylamino-methyl)-3-hydroxy-5-hydroxymethyl-1,2-dimethylpyridinium was dissolved in $10 \mathrm{ml}$ of $\mathrm{HBr}$ (48\%, aq.). The reaction was refluxed for 4 hours. The solvent was removed and the product was obtained. FAB MS: $244.99(\mathrm{M}+)$. ${ }^{1} \mathrm{H}$ NMR $\left(\mathrm{D}_{2} \mathrm{O}, 300 \mathrm{MHz}\right): 8.62$, s, $1 \mathrm{H} ; 4.51, \mathrm{~s}, 2 \mathrm{H} ; 4.24, \mathrm{~s}, 2 \mathrm{H} ; 2.78, \mathrm{~s}, 3 \mathrm{H}$.

\section{4-Aminomethyl-5-dodecylsulfanylmethyl-3-hydroxy-1,2-dimethyl-pyridinium:}

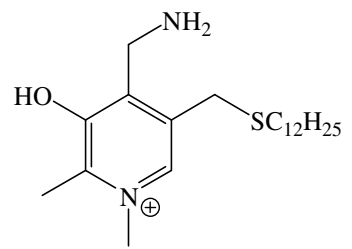

$0.6 \mathrm{ml}$ of 1-dodecanethiol was dissolved in $10 \mathrm{ml}$ of methanol. $10 \mathrm{ml}$ of ammonia aqueous solution (37\%) was added. After the mixture was stirred for 10 minutes, $0.3 \mathrm{~g}$ of 4-aminomethyl-5-bromomethyl-3-hydroxy-1,2-dimethyl-pyridinium was added. The solution was stirred for 30 minutes. The solvent was removed. The product was purified using preparative HPLC. CI MS: $367(\mathrm{M}) .{ }^{1} \mathrm{H}$ NMR $\left(\mathrm{D}_{2} \mathrm{O}\right): 8.21$, s, $1 \mathrm{H} ; 4.49$, s, $2 \mathrm{H} ; 4.20$, s, 3H; 3.97, s, 2H; 2.79, s, 3H; 2.68, t, 2H, J = 7.2 Hz; 1.70, m, 2H; 1.40, m, 18H; 0.99, t, $3 \mathrm{H}, 6.9 \mathrm{~Hz}$. UV-vis: $345 \mathrm{~nm}(\mathrm{pH}=7.5)$.

\section{N,N-Bis-decyl-3-(2,2,8-trimethyl-4H-[1,3]dioxino[4,5-c]pyridin-5-ylmethylsulfanyl)- propionamide:}

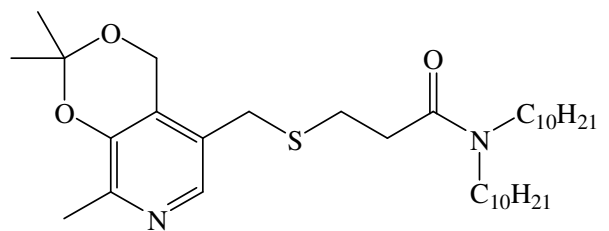

3.9 g 3-[2-(bis-decyl-carbamoyl)-ethyldisulfanyl]-N,N-bis-decyl-propionamide was dissolved in $20 \mathrm{ml}$ of ethanol. $1.5 \mathrm{~g}$ of $\mathrm{NaBH}_{4}$ was added and the mixture was stirred for 20 minutes. Then $2.1 \mathrm{~g}$ of methanesulfonic acid 2,2,8-trimethyl-4H-[1,3]dioxino[4,5c]pyridin-5-ylmethyl ester was added. The reaction was stirred for 1 hour. The product was purified using flash chromatography. ${ }^{1} \mathrm{H} \mathrm{NMR}\left(\mathrm{CDCl}_{3}, 300 \mathrm{MHz}\right): 7.96, \mathrm{~s}, 1 \mathrm{H} ; 5.03$, s, 2H; 3.71, s, 2H; 3.35, m, 4H; 2.89-2.51, m, 7H; 1.79, s, 6H; 1.75-1.23, m, 32H; 1.01, t, $6 \mathrm{H}, \mathrm{J}=6.6 \mathrm{~Hz}$. CI MS: $579(\mathrm{M}+1)$. 


\section{N,N-Bis-decyl-3-(5-hydroxy-4-hydroxymethyl-6-methyl-pyridin-3-ylmethyl-}

sulfanyl)-propionamide:<smiles>CCCCCCCCCCCCCCCCCCCCSCc1cnc(C)c(O)c1CO</smiles>

1.5 g N,N-bis-decyl-3-(2,2,8-trimethyl-4H-[1,3]dioxino[4,5-c]pyridin-5-ylmethylsulfanyl)-propionamide was dissolved in $20 \mathrm{ml}$ of methanol. Then $20 \mathrm{ml}$ of $\mathrm{HCl}$ (aq. conc.) was added. The reaction was stirred overnight. ${ }^{1} \mathrm{H} \mathrm{NMR}\left(\mathrm{CDCl}_{3}, 300 \mathrm{MHz}\right): 7.99$, s, $1 \mathrm{H} ; 5.16, \mathrm{~s}, 2 \mathrm{H} ; 3.87, \mathrm{~s}, 2 \mathrm{H} ; 3.40, \mathrm{~m}, 4 \mathrm{H} ; 2.90-2.59$, m, 7H; $1.95-1.01, \mathrm{~m}, 38 \mathrm{H}$. CI MS: $538(\mathrm{M}+1)$.

N,N-Bis-decyl-3-(4-formyl-5-hydroxy-6-methyl-pyridin-3-ylmethylsulfanyl)propionamide:

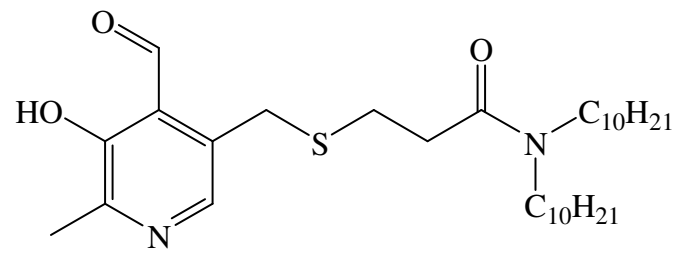

$1.5 \mathrm{~g}$ N,N-bis-decyl-3-(5-hydroxy-4-hydroxymethyl-6-methyl-pyridin-3-ylmethylsulfanyl)-propionamide was dissolved in $50 \mathrm{ml}$ of $\mathrm{CH}_{2} \mathrm{Cl}_{2}$. Then $5 \mathrm{~g}$ of $\mathrm{MnO}_{2}$ was added. The reaction was stirred for 3 hours. ${ }^{1} \mathrm{H}$ NMR $\left(\mathrm{CD}_{3} \mathrm{CN}, 300 \mathrm{MHz}\right): 10.56, \mathrm{~s}, 1 \mathrm{H} ; 8.09$, s, $1 \mathrm{H} ; 4.13, \mathrm{~s}, 2 \mathrm{H} ; 3.30, \mathrm{~m}, 4 \mathrm{H} ; 2.82$, t, $2 \mathrm{H}, \mathrm{J}=6.9 \mathrm{~Hz} ; 2.61$, t, $2 \mathrm{H} ; 2.56, \mathrm{~s}, 3 \mathrm{H} ; 1.70-1.25$, $\mathrm{m}, 32 \mathrm{H} ; 1.01$, t, $6 \mathrm{H}, \mathrm{J}=6.9 \mathrm{~Hz}$. CI MS: $536(\mathrm{M}+1)$. UV-vis $\left(\mathrm{pH}=7.5, \mathrm{CH}_{3} \mathrm{OH}\right): 395 \mathrm{~nm}$. 

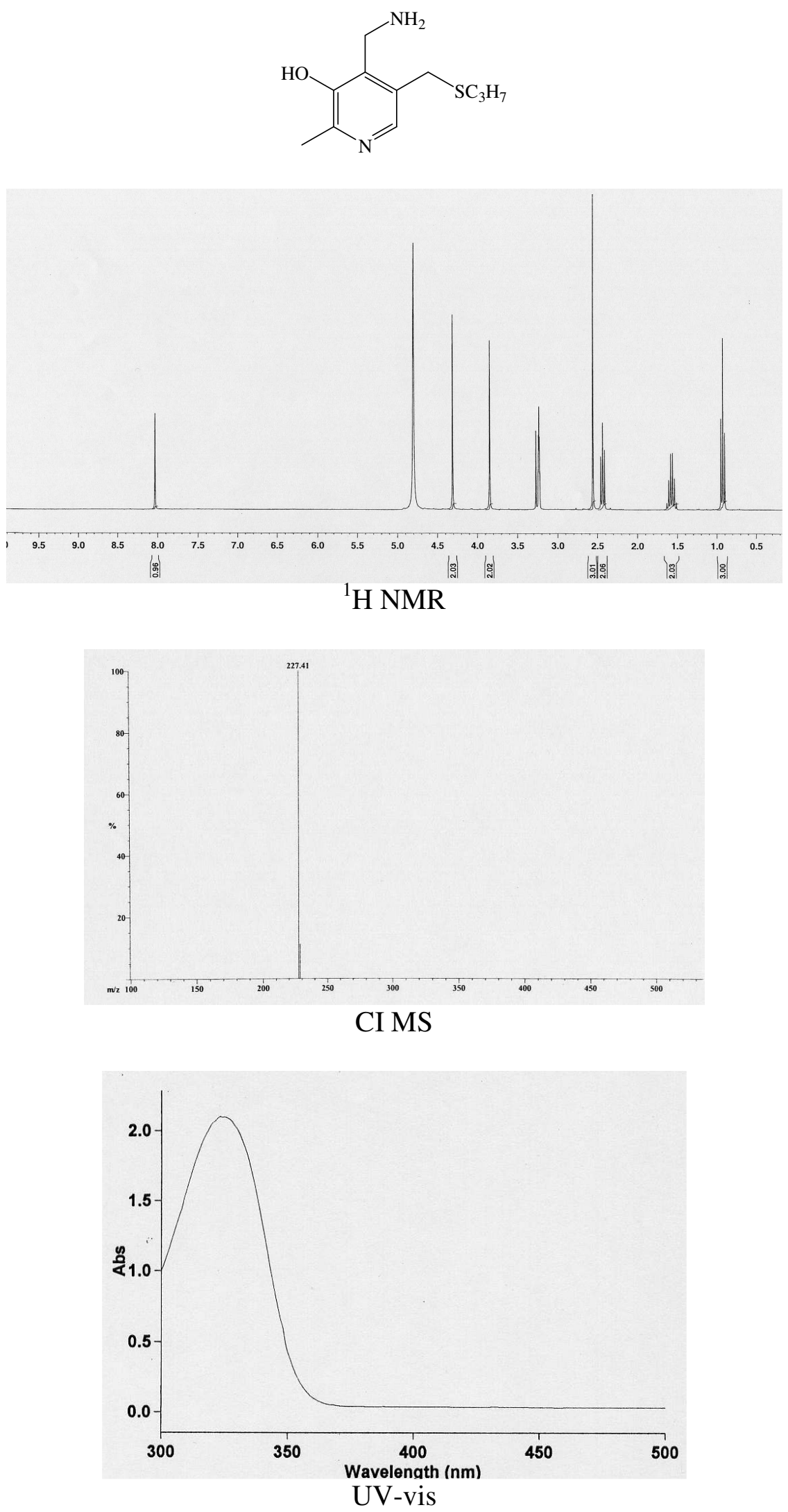

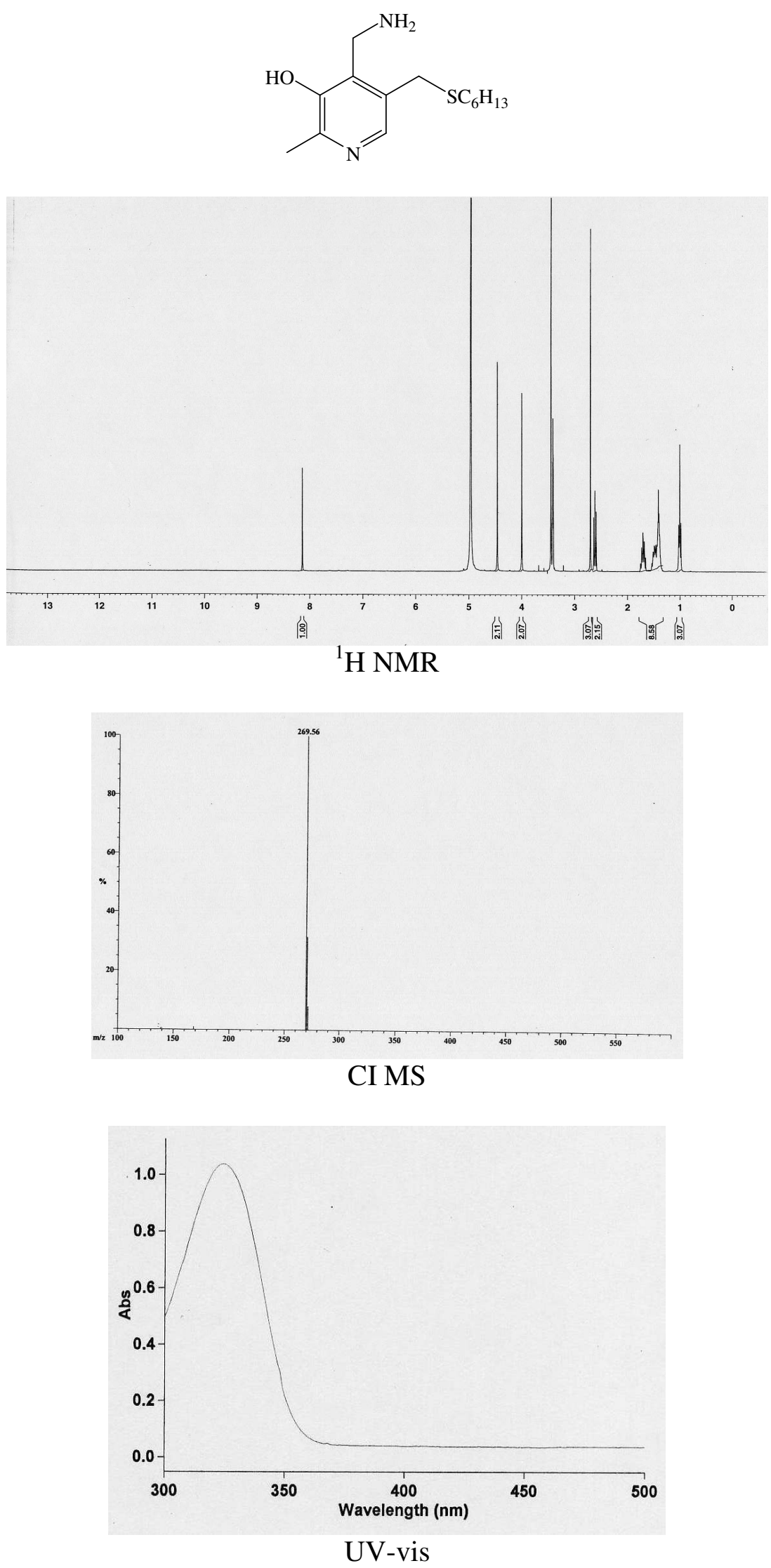

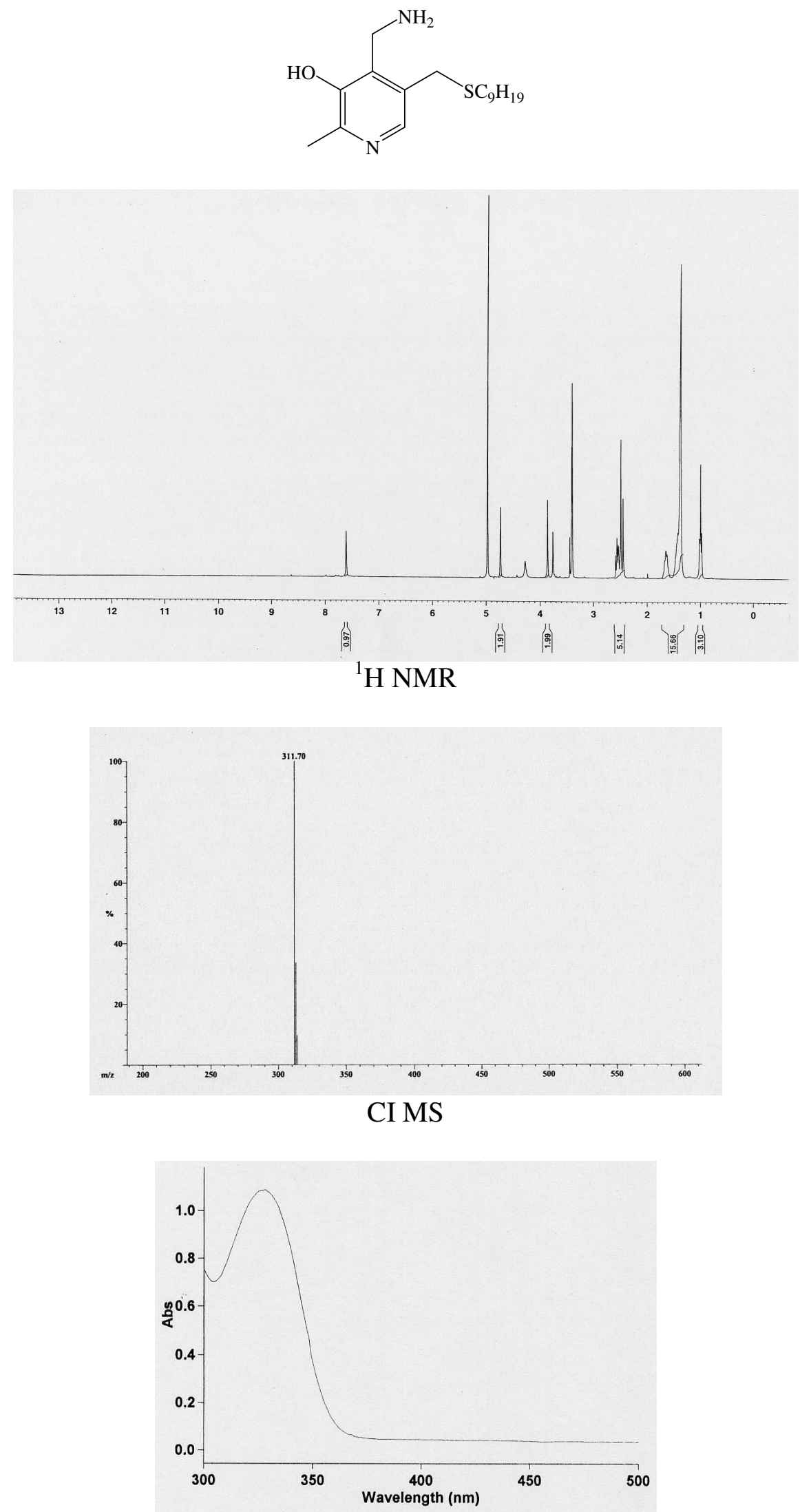

UV-vis 

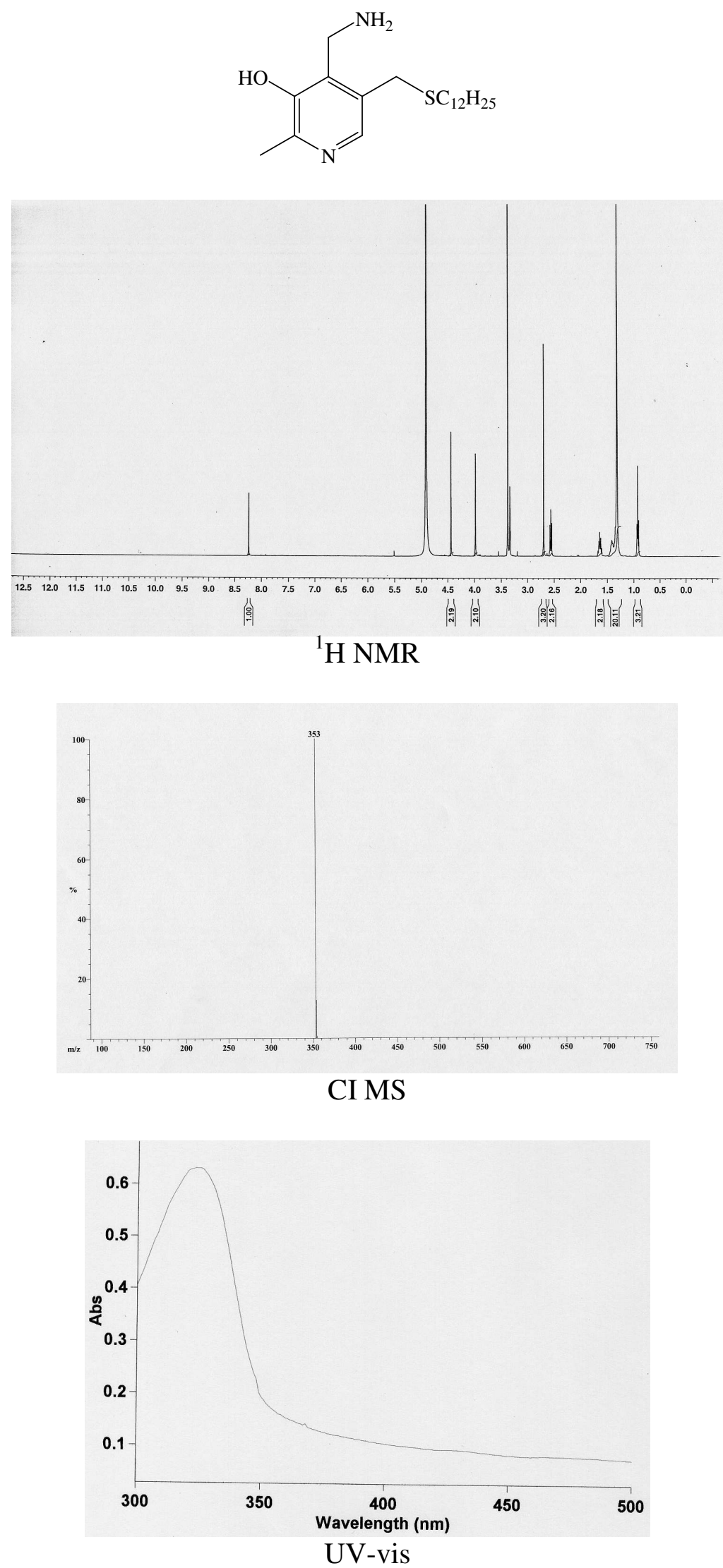

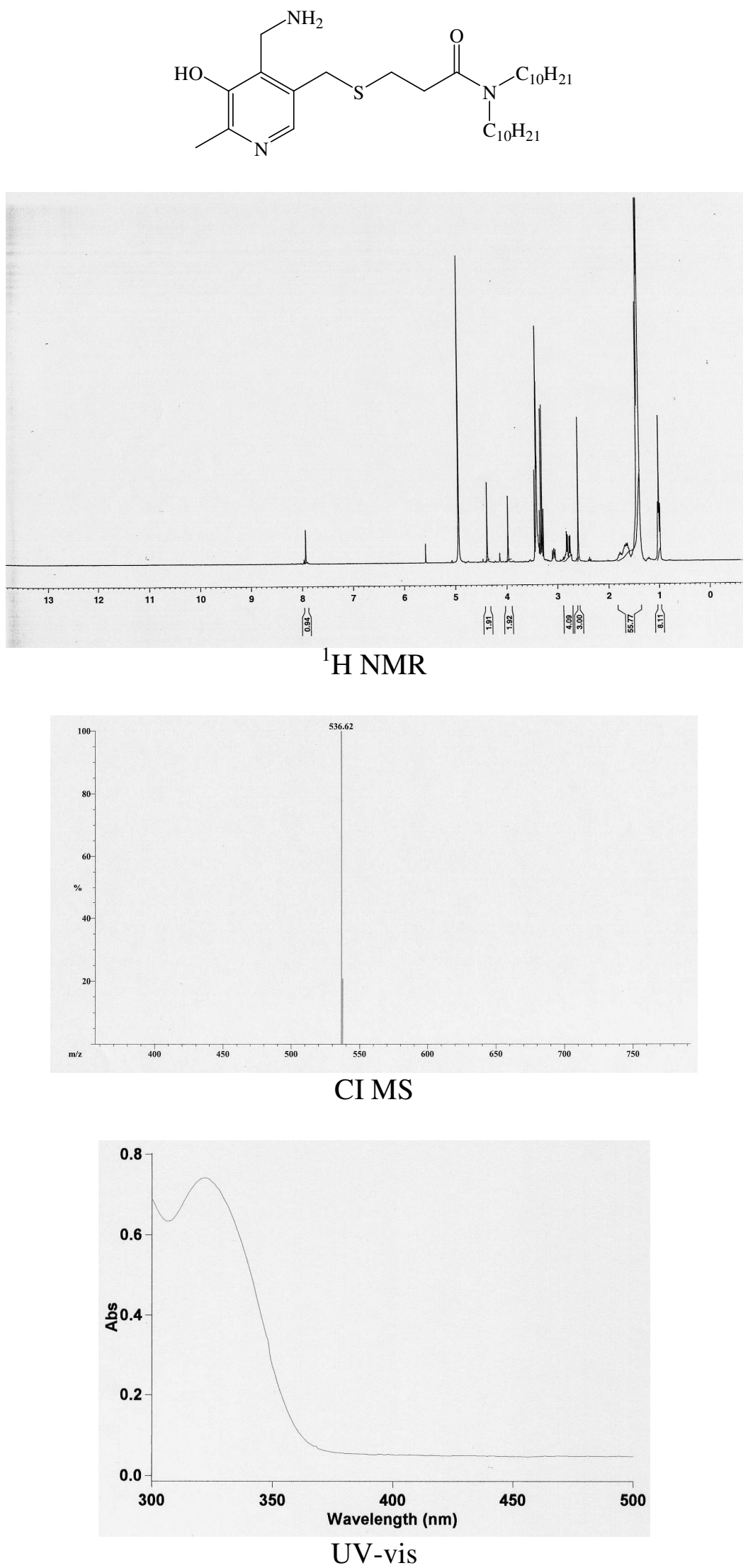

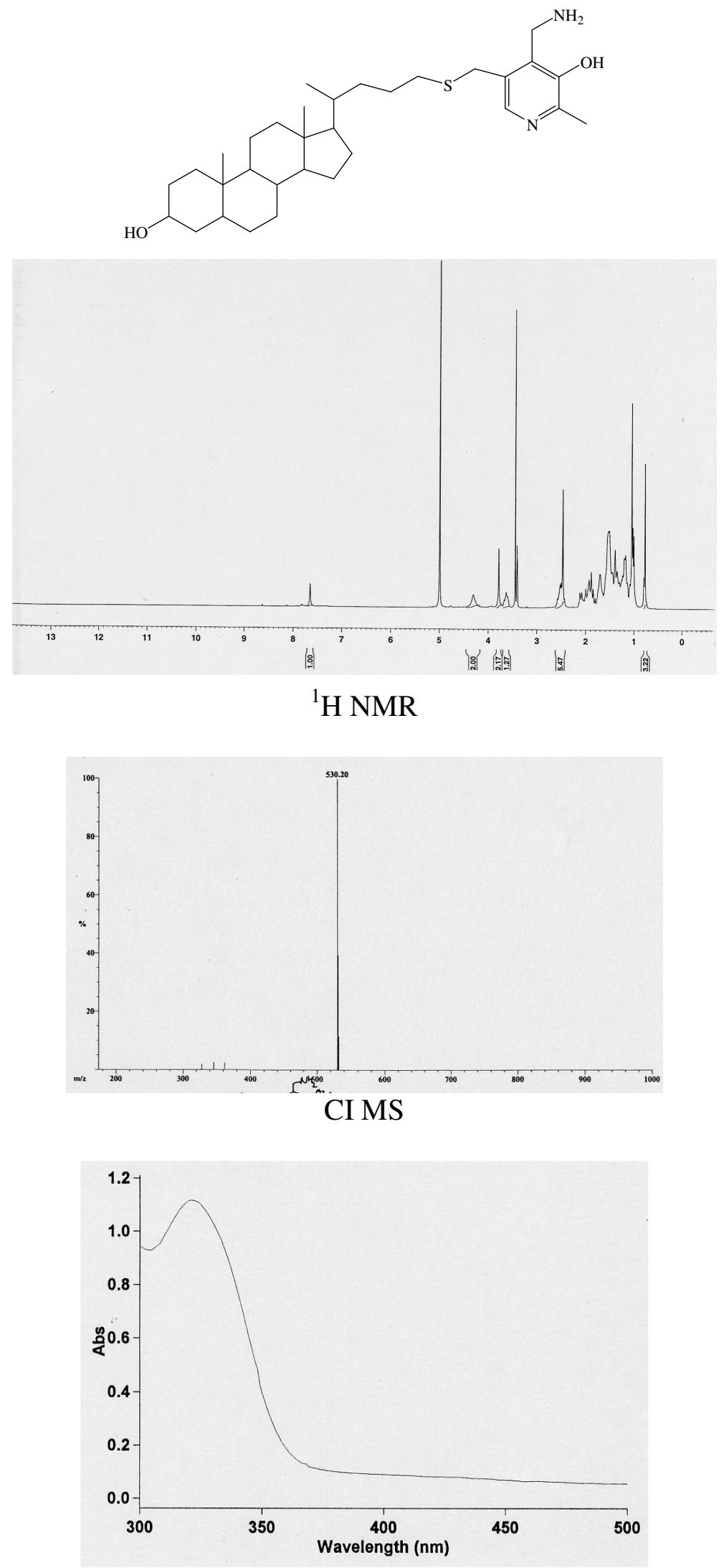

CI MS 

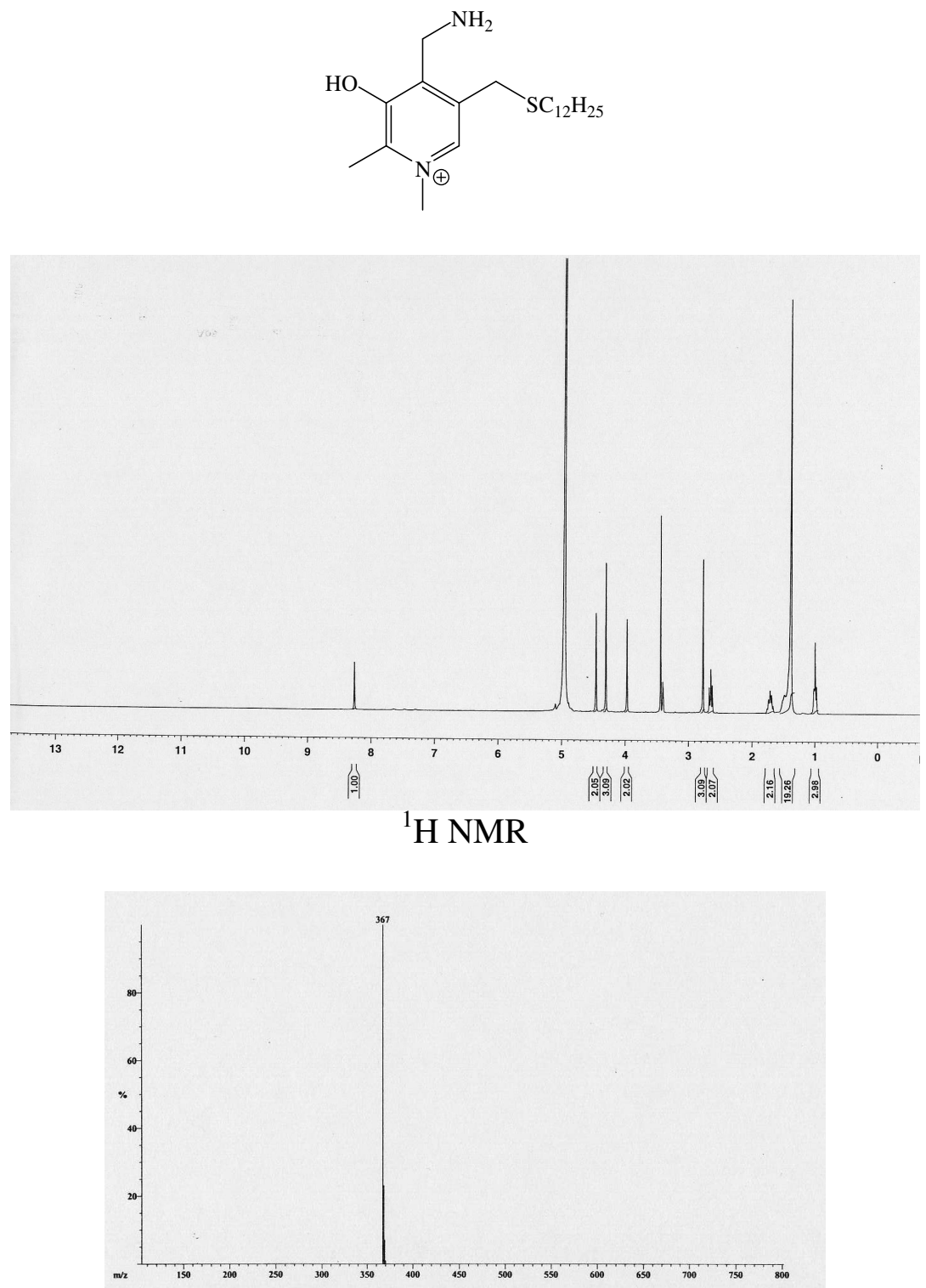

CI MS

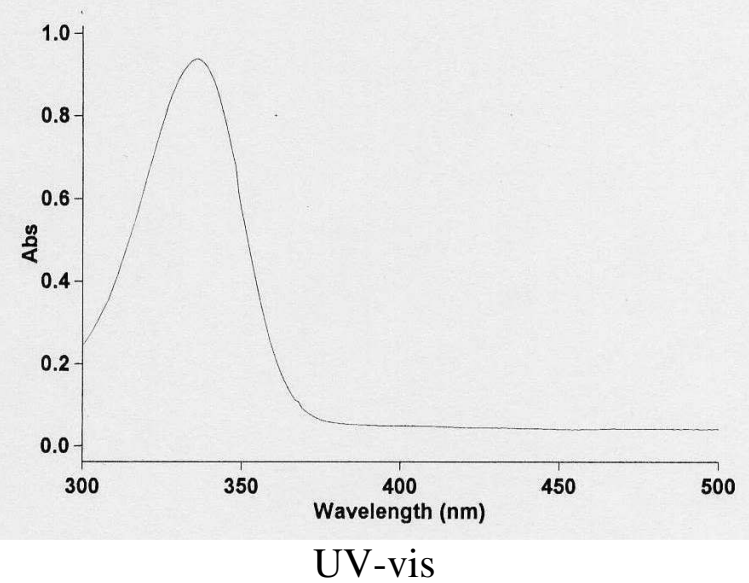




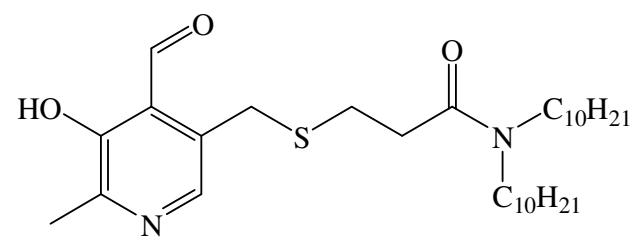
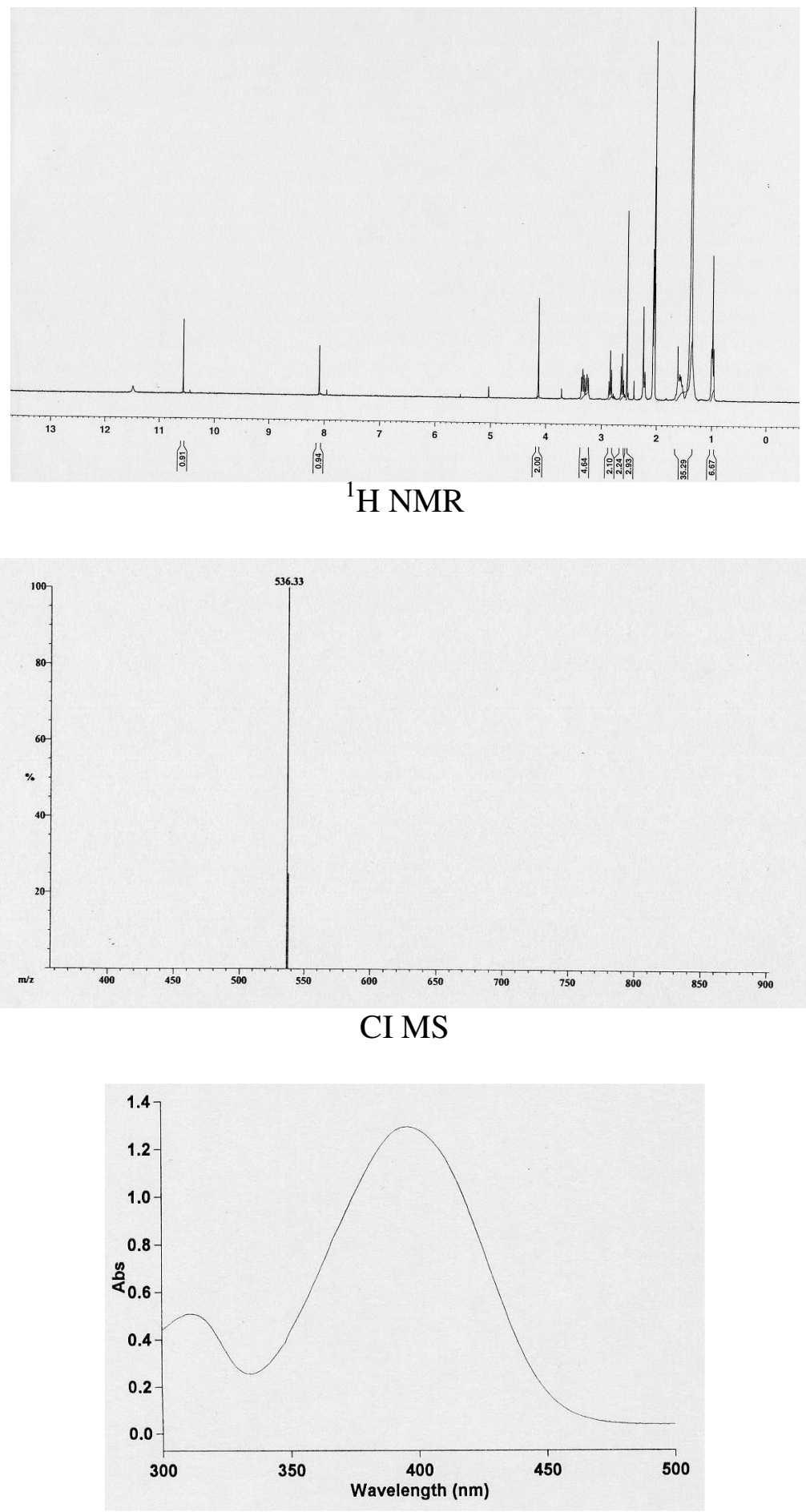

UV-vis 

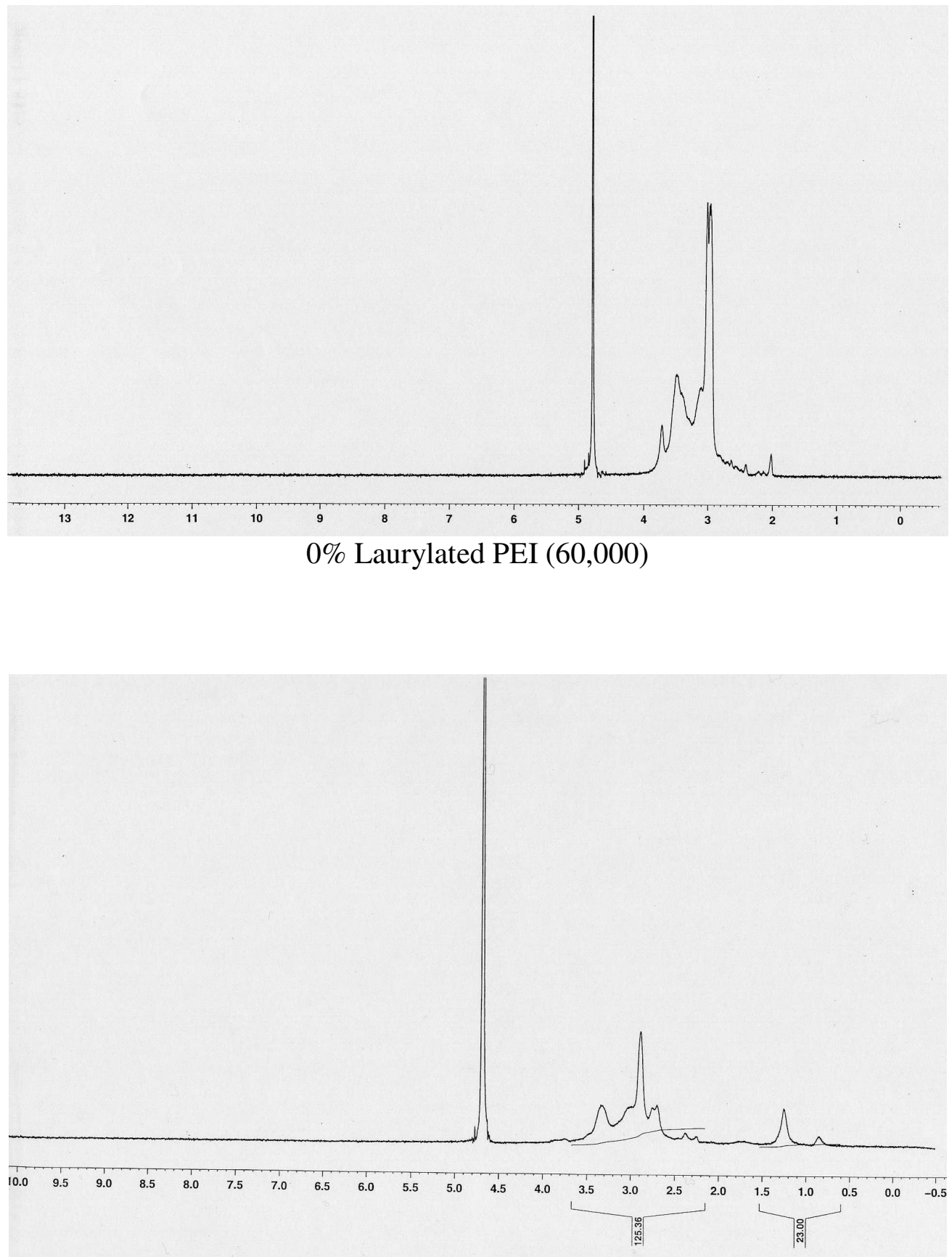

5\% Laurylated PEI $(60,000)$ 


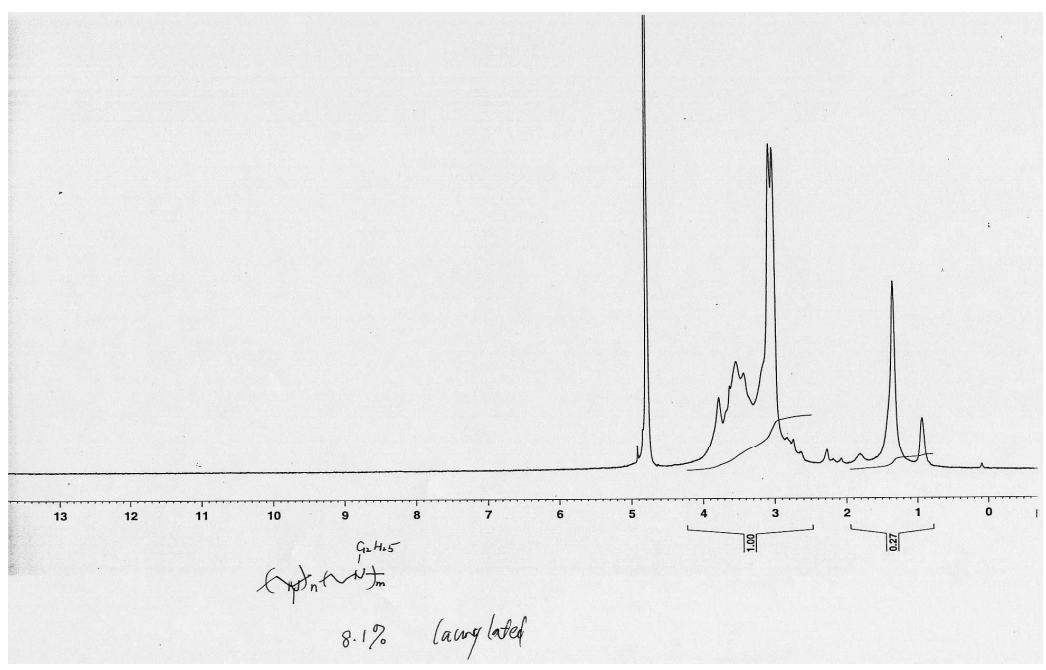

$8 \%$ Laurylated PEI $(60,000)$

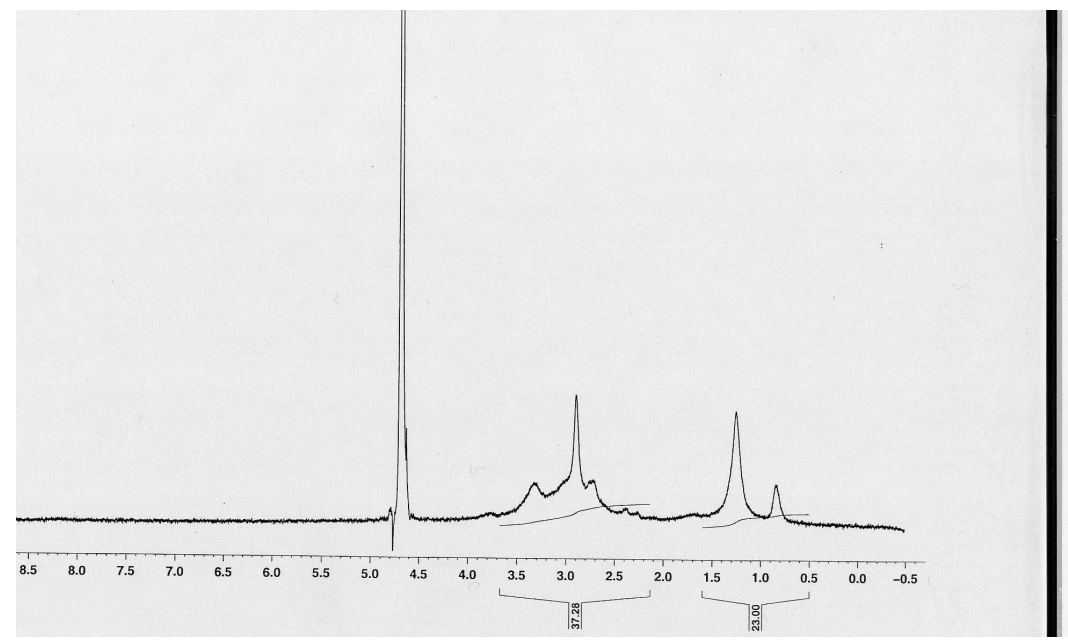

16\% Laurylated PEI $(60,000)$

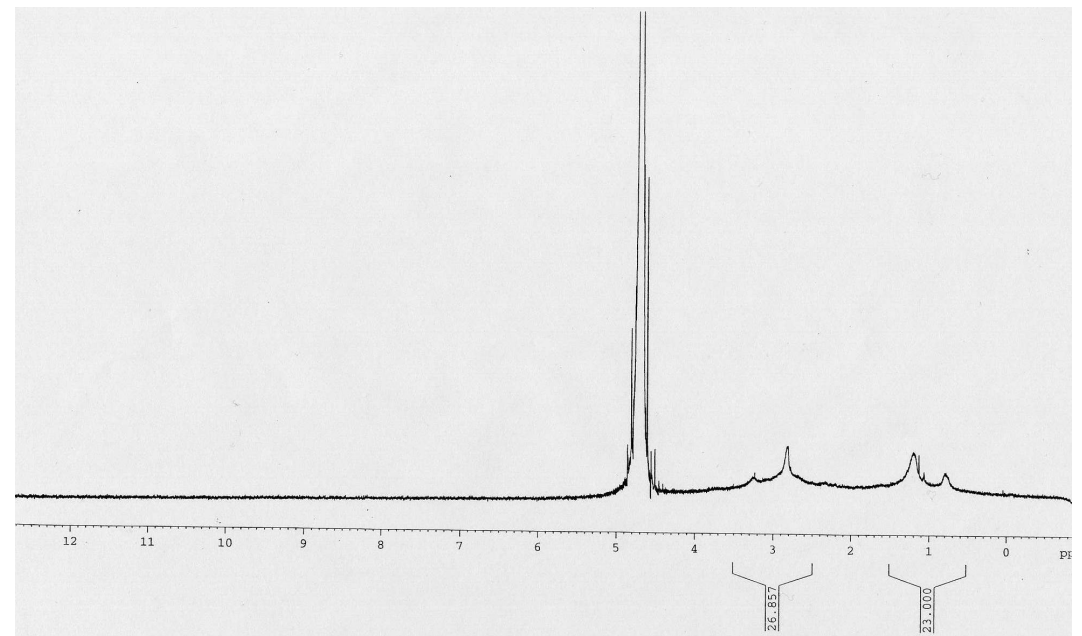

27\% Laurylated PEI $(60,000)$ 


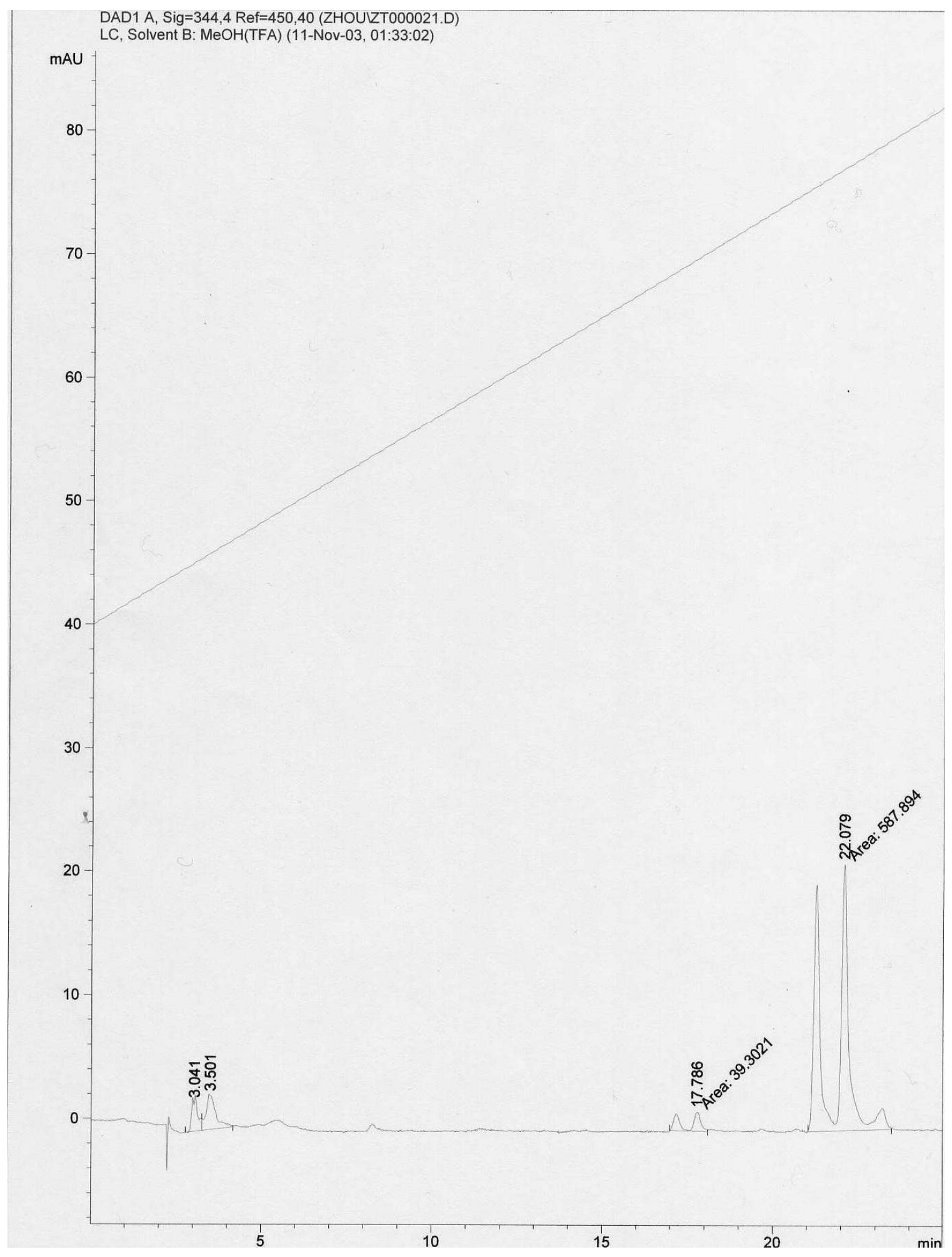

A Typical HPLC Output (L,D-Alanine vs. L,D-Phenylalanine) 